Cinémas

Revue d'études cinématographiques

Journal of Film Studies

\title{
Dagrada, Elena. La Rappresentazione dello sguardo nel cinema delle origini in Europa. Nascita della soggettiva. Bologne : CLUEB, 1998, 339 p.
}

\section{Viva Paci}

Volume 11, numéro 2-3, printemps 2001

Eisenstein dans le texte

URI : https://id.erudit.org/iderudit/024861ar

DOI : https://doi.org/10.7202/024861ar

Aller au sommaire du numéro

Éditeur(s)

Cinémas

ISSN

1181-6945 (imprimé)

1705-6500 (numérique)

Découvrir la revue

Citer ce compte rendu

Paci, V. (2001). Compte rendu de [Dagrada, Elena. La Rappresentazione dello sguardo nel cinema delle origini in Europa. Nascita della soggettiva. Bologne : CLUEB, 1998, 339 p.] Cinémas, 11(2-3), 321-327. https://doi.org/10.7202/024861ar d'utilisation que vous pouvez consulter en ligne.

https://apropos.erudit.org/fr/usagers/politique-dutilisation/ 
DAGRADA, Elena. La Rappresentazione dello sguardo nel cinema delle origini in Europa. Nascita della soggettiva. Bologne: CLUEB, 1998, $339 \mathrm{p}$.

Mi sembrava di vedere tutto attorno visi umani, ma senza gli occhi, e al posto di questi impressionanti cavità nere. "Qua gli occhi, qua gli occhi» gridava Coppelius con voce cupa e tonante.

E.T.A. Hoffmann

Tout au long du siècle dernier, le cinéma a trouvé le temps de devenir autonome, de s'institutionnaliser et de créer ses propres études: de se donner, donc, comme sujet de sa propre théorie, puis de sa propre histoire, puis comme objet d'une sémiologie. Il a créé ses propres études rapidement et de façon déterminée, probablement pour rattraper les "retards", pour ainsi dire, sur les autres domaines d'autorité dans les études académiques. Comme un jeune, en croissance accélérée et en «crise d'adolescence", les études cinématographiques se sont trouvées aux prises avec moult questionnements, questionnements qui déjà avaient été ceux des ses copains plus âgés (notamment l'historiographie et la théorie littéraires). Et comme les études cinématographiques étaient nées à l'époque postfreudienne, les questionnements existentiels de cette crise d'adolescence les ont amenées tout droit vers une très fructueuse, stimulante et longue analyse. Et depuis près de vingt-cinq ans, donc presque un quart de leur jeune existence, les études cinématographiques sont aux prises 
avec de tenaces, patients et méticuleux psychologues! Un chœur se lève, unanime (bénévole, sensible et attentif, bien sûr) pour entonner les diagnostics classiques: "c'est la faute des parents" (les vieux historiens), non pas à cause d'une mauvaise volonté, mais parce qu'eux-mêmes étaient victimes de leurs propres leurres (formes graves de téléologie, syndromes de première fois et "préjugés du précurseur "), "il faut réactiver le refoulé" (rechercher le son du cinéma muet ..., les couleurs dans les pellicules qu'on nous avait conservées en noir et blanc, et des trésors disparus comme le bonimenteur ${ }^{2}$ ), et encore «soyons attentifs au très jeune âge surtout", aux premiers temps. Et en fin de compte: "que les études cinématographiques elles-mêmes apprennent à se poser les bonnes questions». Tout commença, ou bien se déclara, avec le congrès de Brighton en $1978^{3}$... Je voulais, par cette comparaison semi-sérieuse avec l'analyse chez le psy de notre jeune en question, souligner cette série d'amusantes coïncidences et, pour terminer ce parallèle, je me permettrai d'en souligner une dernière. Dans ce domaine, ce n'est pas une découverte isolée qui change soudainement les choses, mais bien les efforts des chercheurs tenaces, patients et méticuleux, disaisje ci-haut, qui rendent la thérapie intéressante et de plus en plus fructueuse. Cela fait donc presque vingt-cinq ans que les études cinématographiques sont en analyse, mais chaque séance permet encore de creuser plus profondément, tout en s'appuyant sur les rencontres précédentes, et s'il y a répétitions, c'est pour le mieux.

La Rappresentazione dello sguardo nel cinema delle origini in Europa. Nascita della soggettiva ${ }^{4}$ d'Elena Dagrada fait justement partie de ces dernières et fructueuses séances d'analyse des études cinématographiques, qui parlent des premiers temps du cinéma, de ses origines. L'étude sur la représentation du regard au cinéma, des fonctions de cette représentation, de l'origine de la forme (sources iconographiques et thématiques) avait déjà fait l'objet de plusieurs articles de Dagrada ${ }^{5}$. Cet intéressant ouvrage s'articule en deux parties. Dans la première, l'auteure, consciente et convaincue du fait que le cinéma est "le produit d'une culture et de son histoire" (p. $7^{6}$ ), se pose une question: d'où vient-il, le plan subjectif? c'est-à-dire, de façon plus détaillée, 
"d'où viennent-elles et comment s'imposent-elles, ces formes de l'image cinématographique qui signifient, pour le spectateur d'aujourd'hui, la présence d'un personnage qui regarde?» (p. 7). Déjà, dès les toutes premières pages de l'introduction, l'auteure fait preuve d'une très rassurante cohérence méthodologique, et ne laisse rien au hasard. Ainsi, ce qui en français cinématographique courant s'appellerait caméra subjective (depuis Jean Mitry, 1965) ou plan subjectif (depuis Christian Metz, 1971 et 1972), dans la suite de son ouvrage s'appellera représentation $d u$ regard. Car, comme l'école gaudreaultienne le souligne', il faut être très attentifs à l'égard des mots qui dans l'usage courant, avec les connotations acquises, indiquent un univers qui ne correspond pas à celui des premiers temps du cinéma :

\begin{abstract}
Parler de plan subjectif, en effet, signifie présupposer $\grave{a}$ tout le moins les concepts de personnage et de diégèse, puisque [...] le plan subjectif se caractérise en tant que mise en scène du regard d'un personnage, en tant que regard diégétique ${ }^{8}$. Or, dans le cinéma des origines manquent soit la constitution de l'acteur en personnage, soit la mise en scène d'un univers diégétique clos et structuré selon les lois du récit cinématographique, classique, moderne ou contemporain. Mieux, nous manquent les conditions pour pouvoir assimiler aisément ce qu'on entend aujourd'hui quand on parle de plan subjectif, de personnage, de diégèse et de récit cinématographique, aux phénomènes apparemment analogues du cinéma des origines (p. 8).
\end{abstract}

De plus, l'expression plan subjectif (soggettiva en italien, chez Dagrada) «suggère la superposition d'une fonction expressive et psychologique et d'une autre plus proprement perceptive et linguistique» (p. 30). Ces considérations de méthode, que pose comme prémisses l'examen plus spécifique des films européens de la période 1896-1910, renforcent son ouvrage tout au long, selon un absolu bon sens. Sa démarche est claire: pour expliquer un élément (seulement apparemment) proprement cinématographique, la représentation du regard, Dagrada recherche dans une plus vaste histoire de la vision, davantage depuis le XVIII ${ }^{e}$ siècle, la naissance et le développement de cette forme de l'image qui 
signifie pour un spectateur qu'il se trouve en présence d'un personnage qui regarde. Un peu à la façon de Leutrat qui, dans le premier chapitre de Le Cinéma en perspective (1992), nous propose une histoire de la vision assez personnelle et fait un panorama de ces phénomènes ou de ces séries culturelles qui d'après lui ont des affinités avec le cinéma en mettant en évidence les déplacements de la perception qui s'effectuent entre le XVIII et le XIX siècle, Dagrada s'appuie sur les merveilleuses études, entre autres, de Max Milner (1982) et de Wolfgang Schivelbusch (1977) pour peindre un univers où le regard, le regard mobile et un certain voyeurisme ont une place déterminante dans l'histoire des mœurs et de la culture. La littérature fantastique, qui travaille sur l'invention de nouvelles modalités de regarder et de laisser voir, dans le but de satisfaire la pulsion scopique qui est à la base de tout travail d'imagination, et l'invention des voyages en chemin de fer, qui contribue au changement épistémologique de l'idée de vision à la fin du XIX siècle ${ }^{9}$, sont des composantes importantes de ce regard, dans le fond un regard virtuel ${ }^{10}$, dont Elena Dagrada s'occupe.

Cette première partie de l'ouvrage nous présente un très riche parcours parmi les variations (en suivant un chemin davantage chronologique) de la représentation du regard dans les films. Généralement, l'articulation évolutive (sans téléologie, bien sûr!), proposée par Gunning et Gaudreault ", de "système d'attractions monstratives " à "système d'intégration narrative", est respecté dans l'analyse des cas que Dagrada présente (symptomatique, le titre du troisième chapitre: "Vers la linéarisation"). Au fil du texte, une multitude de titres du cinéma des origines européen sont continuellement proposés comme preuves du vaste répertoire des possibilités de manifestations que la représentation du regard au cinéma peut assumer.

La deuxième partie du livre fournit une série de fiches filmographiques conçues par l'auteure pour articuler son discours. Les fiches (en ordre alphabétique par titre de film, mais, bien sûr, une introduction nous précise les choix effectués dans cet archivage, surtout dans les cas des fréquentes exceptions) présentent comme d'habitude une partie technique, le pays d'origine et la cinémathèque ou archive dépositaire, en plus d'une description 
détaillée du plan (ou des plans dans les cas de vues pluriponctuelles) et des éventuels mouvements de caméra.

À ces deux parties s'ajoute une petite section iconographique (œuvres - toiles, figurines, étampes, vitres de projection, illustrations, etc. - entre le XVII et le début du XXe siècle) dont le regard (et le regardant et le regardé) est la vedette.

Cet ouvrage concilie donc la rigueur de la compilation des fiches filmographiques à une première partie très riche de références variées, et toujours pertinentes. De plus la forme de l'écriture est recherchée et agréable.

Il s'agit d'un livre très intéressant aussi du point de vue didactique. Cet ouvrage rappelle, commente, met en perspective et interroge parfois les théories contemporaines majeures du cinéma, en reconstituant les relations, les parrainages, les petites disputes de famille... entre les nouveaux historiens qui s'occupent du cinéma des origines. Ce qui est fort formateur pour le chercheur qui n'a pas participé, pendant les vingt dernières années aux colloques et aux festivals voués au cinéma des origines. La richesse des références aux incontournables de la nouvelle histoire du cinéma, et un bon nombre d'ouvertures moins prévisibles, mais très appropriées et pertinentes (Schivelbush et Milner), fournissent une rampe d'accès fort utile pour s'initier aux problèmes de l'histoire du cinéma et aux questions de méthode que ceux-ci imposent, et dont le cinéma des origines constitue un cas si exemplaire. Intéressant du point de vue didactique, donc, car ce cadre stimulant est une très bonne façon, entre autres, de conscientiser l'étudiant en cinéma à une juste, créative (tout en restant bien documentée) problématisation des cas qu'il rencontre... On en attend donc une bonne traduction en français.

Un livre moderne d'histoire du cinéma, où histoire et théorie s'entremêlent et s'interrogent. La Rappresentazione dello sguardo nel cinema delle origini in Europa. Nascita della soggettiva fourmille d'observations tout à fait d'actualité et d'analyses très finement menées. Les questions reliées au cinéma des origines n'ont pas fini de donner de la matière de travail au chercheur et ce livre, consacré à l'étude d'un aspect particulier bien centré et paradigmatique, a le mérite d'avoir reproposé très clairement et dynamiquement des jalons à partir desquels la réflexion, déjà 
bien entamée, pourra se poursuivre, avec en plus à son actif de nouvelles acquisitions sur une figure, celle du regard, si fondamentale au cinéma.

Viva Paci

Université de Montréal

\section{NOTES}

1. Je reprends l'expression "préjugés du précurseur" d'Elena Dagrada qui précise: " [...] tendance à juger l'œuvre d'un cinéaste sur des critères qui n'appartiennent pas à son temps, mais au nôtre, et selon laquelle la valeur dépend directement du fait d'avoir su ou non anticiper notre présent" (p. 39) (c'est moi qui traduis). Pour ce qui est de ces leurres, la bibliographie est, aujourd'hui, énorme. Je me limite à rappeler la première fois (sic!) que ces préoccupations ont été l'objet d'une réflexion plus méthodique. Il s'agit de la fameuse série d'articles de Jean-Louis Comolli, publiée dans les Cahiers du Cinéma ( $\left.{ }^{\text {os }} 229-231,233,1971 ; n^{\text {os }} 234-235,1971 / 1972 ; n^{\circ} 241,1972\right)$.

2. Je ne signalerai que deux textes à ce sujet. Le premier, parce que le titre tombe fort bien à propos: "Le retour du [bonimenteur] refoulé ... ", d'André Gaudreault (collaboration: Jean-Pierre Sirois-Trahan, Iris, $\mathrm{n}^{\circ} 22,1996$, p. 17-32); et l'autre, parce que de toute récente parution et constituant une bonne mise au point sur la question: Le Bonimenteur de vues animées, de Germain Lacasse (Paris/Québec: Méridiens Klincksieck/Nota Bene, 2000).

3. On a l'habitude de nommer "nouveaux" une certaine classe d'historiens, essentiellement ceux qui participèrent au fameux congrès de la FLAF tenu à Brighton en 1978, où l'on présenta plus de six cents films, pour la plupart inédits, de la période entre 1900 et 1906, donnant ainsi les outils jusqualors inaccessibles pour tout un champ de réflexion. Les actes de ce congrès ont été publiés en deux recueils: Roger Holman (direction), Cinema 1900-1906: An Analytical Study, vol. I (Bruxelles: FIAF, 1982), qui présente les communications et les discussions qui accompagnèrent le visionnement des films; et André Gaudreault (direction), Cinema 1900-1906: An Analytical Study, vol. II, Filmography (Bruxelles: FIAF, 1982), qui propose une filmographie analytique des films présentés au congrès. Depuis, une nouvelle conscience permit de ne plus regarder le cinéma des origines comme un noyau dans lequel étaient contenus les éléments du cinéma futur pas encore développés à cause des pauvres capacités techniques que l'immaturité du moyen consentait. Le renouveau du regard des historiens a en effet permis de voir ce cinéma-là comme quelque chose de différent des développements futurs du cinéma: un cinéma autre, avec une autre façon d'être montré et une autre façon d'être regardé. Pour un bilan de la nouvelle histoire du cinéma, en particulier appliquée au cinéma des premiers temps, cf. André Gaudreault et Denis Simard, "L'extranéité du cinéma des premiers temps: bilan et perspectives de recherche" (dans Jean A. Gili, Michèle Lagny, Michel Marie et Vincent Pinel (direction), Les vingt premières années du cinéma français, Paris: Presses de la Sorbonne Nouvelle et AFRHC, 1995, p. 15-28). Cf. aussi Jacques Aumont, André Gaudreault et Michel Marie (direction), Histoire du cinéma. Nouvelles approches (Paris: Publications de la Sorbonne, 1989).

4. La Représentation du regard dans le cinéma des origines en Europe. Naissance du plan subjectif (ma traduction).

5. Voir à ce sujet les articles de Dagrada: "Sulla soggettiva libera indiretta ", $C i$ nema \& Cinema, $\mathrm{n}^{\circ} 43$ (1985); "The Diegetic Look. Pragmatics of the Point-of-View Shot ", Iris, $\mathrm{n}^{\circ} 7$ (1986); "Subjectivité et caméra subjective", Actes sémiotiques, vol. X, 
n" 41 (1987); "Un regard indiscret - caméra subjective et cinéma des premiers temps", dans Ancré Gaudreault (direction), Ce que je vois de mon ciné (Paris : Méridiens-Klincksieck, 1988); "Through the Keyhole", Iris, n 11 (1990).

6. C'est moi qui traduis toutes les citations du livre de Dagrada.

7. Voir à ce sujet, particulièrement, André Gaudreault, "Les vues cinématographiques selon Georges Méliès, ou : comment Mitry et Sadoul avaient peut-être raison d'avoir tort (même si c'est surtout Deslandes qu'il faut lire et relire) ", dans Jacques Malthête et Michel Marie (direction), Georges Méliès, l'illusionniste fin de siècle? (Paris: Presses de la Sorbonne Nouvelle, 1997). Par rapport aux "modes d'emploi" lexicaux que Gaudreault suggère, un seul écart remarquable se présente dans le texte de Dagrada. Notre auteure appelle ses objets "films des origines" (film delle origini en italien) ou parfois "petits films" (filmetti en italien), et surtout plus souvent carrément films; sans considérer donc le conseil encore gaudreaultien de les appeler vues, pour nous aider, par le biais d'un choix lexical, à comprendre et à se souvenir du fait que le cinéma des premiers temps est étranger au cinéma d'après l'institutionnalisation (date quelque peu variable, on le sait, selon les théoriciens-historiens, mais q!ıi à quelques années près chevauche les années dix). "[...] utilisation systématique des termes et expressions en usage à l'époque du cinéma des premiers temps. Dire par exemple "fabrication de vues animées" plutôt que "production cinématographique", "cinématographiste" plutôt que "cinéaste", "vue" plutôt que "film", etc." (p. 113). Et encore Gaudreault et Simard, op. cit. : "Nous parlons d" extranéité" plutôt que d" étrangeté" tout simplement parce que ce n'est pas le fait que le cinéma des premiers temps soit étrange que nous voulons mettre en relief, mais plutôt le fait qu'il soit étranger. Le cinéma des premiers temps est irréductiblement étranger au cinéma qui l'a suivi, et le cinéma des premiers temps est irréductiblement étranger au spectateur en différé que nous sommes" (p. 22).

8. Voir à ce sujet Dagrada (1986).

9. De plus, le chemin de fer naissant, de même que l'électricité et les architectures en verre permettent à l'œil de voir ce qui jamais n'avait été donné à voir: voir dans l'obscurité, voir à travers, voir en mouvement ...

10. Sur l'idée de regard virtuel, des pages intéressantes se trouvent dans le texte d'Anne Friedberg, Window Shopping. Cinema and Postmodern (Berkley/Los Angeles/Oxford: University of California Press, 1993). Friedberg parle de mobilized virtual gaze. Virtuel et mobile, car il s'agit ici d'un regard dont la perception est médiatisée par une représentation; le cinéma depuis son invention avait pu combiner le mobile et le virtuel.

11. Cf. André Gaudreault et Tom Gunning, "Le cinéma des premiers temps: un défi à l'histoire du cinéma?", dans Jacques Aumont, André Gaudreault et Michel Marie (direction), Histoire du cinéma. Nouvelles appraches (Paris: Publications de la Sorbonne, 1989), p. 49-63.

\section{OUVRAGES CITÉS}

Leutrat, Jean-Louis. Le Cinéma en perspective: une histoire. Paris : Nathan, 1992.

Metz, Christian. Langage et cinéma. Paris: Larousse, 1971.

Metz, Christian. Essais sur la signification au cinéma, Tome II. Paris: Klincksieck, 1972.

Milner, Max. La Fantasmagorie. Essai sur l'optique fantastique. Paris: PUF, 1982.

Mitry, Jean. Esthétiçue et psychologie du cinéma, Tome II. Paris: Éditions universitaires, 1965.

Schivelbusch, Wolfgang. Geschichte der Eisenbahnreise. München/Wien : Carl Hanser Verlag, 1977. 